\title{
Suppression of molecular decay in ultracold gases without Fermi statistics
}

\author{
J. P. D'Incao ${ }^{1,2}$ and B. D. Esry ${ }^{2}$ \\ ${ }^{1}$ Department of Physics and JILA, University of Colorado, Boulder, Colorado 80309-0440, USA \\ ${ }^{2}$ Department of Physics, Kansas State University, Manhattan, Kansas 66506, USA
}

\begin{abstract}
We study inelastic processes for ultracold three-body systems in which only one interaction is resonant. We have found that the decay rates for weakly bound molecules due to collisions with other atoms can be suppressed not only without fermionic statistics but also when bosonic statistics applies. In addition, we show that at ultracold temperatures three-body recombination involving a single resonant pair of atoms leads mainly to formation of weakly bound molecules which, in turn, are stable against decay. These results indicate that recombination in three-component atomic gases can be used as an efficient mechanism for molecular formation, allowing the achievement of high molecular densities.
\end{abstract}

PACS numbers: 34.50.-s,34.50.Cx,67.85.-d,31.15.x

In recent years, the efficiency of diatomic molecule formation in ultracold quantum gases and their stability once formed have become key ingredients for many experiments. For example, the remarkable stability of weakly bound molecules in ultracold gases of fermions in two different spin states [1] has greatly helped experimental studies in the BEC-BCS crossover regime [2] when tuning the two-body $s$-wave scattering length $a$ from $a>0$ to $a<0$ near a Feshbach resonance. The long lifetimes recently observed for ultracold heteronuclear molecules [3] might also pave the way for future studies on ultracold polar molecules. The stability of weakly bound molecules where at least one atom is a fermion has been interpreted as a result of suppression due to the fermionic statistics governing both atom-molecule and moleculemolecule collisions [4, 5]. When bosonic statistics plays a role, however, weakly bound molecules tend to decay rapidly, limiting the experimental possibilities for studies near a Feshbach resonance [5, 6, 7]. Obviously, besides stability, efficient production of molecules is also of crucial experimental importance.

In this Letter, we present a series of new predictions that opens up new possibilities for obtaining long molecular lifetimes and that also provide an alternative path for efficient molecular formation. Surprisingly, we have found that weakly bound molecules of two resonantly interacting atoms can still be stable against collisions with other atoms even when bosonic statistics applies. Specifically, inelastic collisions between these weakly bound molecules and any atom not resonant with either of the molecule's atoms is suppressed as $a^{-1}$, therefore allowing long molecular lifetimes near a Feshbach resonance. This suppression in the absence of fermionic statistics has recently been observed in Ref. [3] in an ultracold mixture of weakly bound ${ }^{87} \mathrm{Rb}^{40} \mathrm{~K}$ molecules and ${ }^{40} \mathrm{~K}$ atoms in an nonresonant state. Here, we show that the mechanism that determines the $a^{-1}$ suppression can be traced to the Efimov physics governing the atom-molecule interactions [5]. In contrast to the cases with fermionic suppression [4, 5], our predicted $a^{-1}$ suppression holds for any mass ratio between the collision partners. The sole requirement is that only the atoms bound in the molecule interact resonantly.

We will also show that three-body recombination in such systems displays interesting and potentially useful behavior. In the one- and two-component bosonic gases and in bose-fermi mixtures studied to date, recombination can lead to a substantial fraction of deeply bound molecules [5, 8, 9], giving them in the process enough kinetic energy to escape from typical traps. Moreover, any weakly bound molecule formed can eventually decay rapidly due to collisions with other atoms and molecules 10]. The situation is better for fermionic gas mixtures of two spin species: molecules are stable [4, 5] and the recombination rate into weakly bound molecules $\left(\propto T a^{6}\right)$ dominates recombination into deeply bound molecules $\left(\propto T a^{2.455}\right)$ [5]. These two ingredients ensured the achievement of highly efficient molecular formation and long molecular lifetimes [11]. However, because recombination for fermionic systems scales as $T a^{6}$, efficient molecule formation is restricted to relatively high temperatures or large scattering lengths [1]. In this regime, though, collisions can potentially lead to high molecular dissociation rates $\left(\propto T^{3} a^{6}\left[\begin{array}{l}5 \\ ]\end{array}\right)\right.$, limiting the molecular lifetimes.

As we will show, a three-component gas of atoms with only a single resonant interaction retains the advantages of these fermionic systems - efficient formation of weakly bound molecules and long lifetimes with the additional advantage of having a non-zero recombination rate at $T \rightarrow 0$, allowing the formation of molecules with much lower temperature which is important to explore the quantum degenerate regime. We have found that for these systems three-body recombination into weakly bound molecules scales as $a^{4}$ at ultracold temperatures while recombination into deeply bound molecules scales as $a^{2}$. This weaker $a$-dependence ensures that near a Feshbach resonance recombination will lead mainly to formation of weakly bound molecules. As shown in Refs. [10, 11], these weakly bound molecules 
can stay trapped due to the small energy released, minimizing atomic and molecular losses. The most important aspect of molecular formation using this mechanism, however, is that even in the absence of fermionic statistics, the molecules formed are stable against decay since the decay rate scales as $a^{-1}$. This combination of higher formation rate and lower decay rate maximizes molecule production.

We study these ultracold three-body collisions by solving the Schrödinger equation in the adiabatic hyperspherical representation. The three-body inelastic collision rates are determined by solving the hyperradial Schrödinger equation given by (in atomic units)

$$
\left[-\frac{1}{2 \mu} \frac{d^{2}}{d R^{2}}+W_{\nu}\right] F_{\nu}+\sum_{\nu^{\prime} \neq \nu} V_{\nu \nu^{\prime}} F_{\nu^{\prime}}=E F_{\nu}
$$

Here, $\mu$ is the three-body reduced mass, $E$ is the total energy, $F_{\nu}$ the hyperradial wave function, and $\nu$ a collective index that represents all quantum numbers necessary to label each channel. Since the hyperradius $R$ gives the overall size of the system, this equation describes the collective radial motion under the influence of the effective potential $W_{\nu}$, with inelastic transitions driven by the nonadiabatic couplings $V_{\nu \nu^{\prime}}$ [5].

In the regime where the two-body interactions are resonant, i.e., when $|a| \gg r_{0}$ (where $r_{0}$ is the characteristic range of two-body interactions), the effective potentials $W_{\nu}$ become universal, and several analytical properties of ultracold three-body systems can be derived. In fact, in our framework [5] both the $a$ and the $E$ dependence of the three-body collision rates can be determined by simply identifying the attractive or repulsive character of $W_{\nu}(R)$ as determined by the influence of Efimov physics [5]. Previous work in ultracold three-body collisions [4, 5, 7, 9] has analyzed the influence of Efimov physics for systems where at least two of the pairwise two-body interactions are resonant. In this paper, we will show that Efimov physics still has an important impact even when just one interaction is resonant.

For systems with single resonant interaction, the effective potentials are repulsive in the range $r_{0} \ll R \ll|a|$ and can be conveniently parametrized by the coefficients $p_{0}$ and $p_{\nu}$ [5]:

$$
W_{0}(R)=\frac{p_{0}^{2}-\frac{1}{4}}{2 \mu R^{2}} \quad \text { and } \quad W_{\nu}(R)=\frac{p_{\nu}^{2}-\frac{1}{4}}{2 \mu R^{2}} .
$$

For $a>0, p_{0}$ is associated with the atom-dimer channel and therefore plays an important role in atom-molecule processes. The effective potentials associated with $p_{\nu}$ describe three-body continuum channels. The coefficients $p_{0}$ and $p_{\nu}$ in Eq. (2) can be determined by assuming a zero-range model potential for the interatomic interactions (we have confirmed that finite-range two-body interactions give the same results) and writing the threebody wave function in terms of the Faddeev components
[5]. Doing this, we have determined that $p_{0}$ and $p_{\nu}$ are

$$
p_{0}=J+1, \quad p_{\nu}=J+3, J+5, \ldots
$$

where $J$ is the total orbital angular momentum. Interestingly, in contrast to systems where two or three interactions are resonant, the strengths of the potentials $p_{0}$ and $p_{\nu}$ do not depend on either the mass ratio or the permutational symmetry of the resonant pair.

Our derivation of these rates' scaling behavior rests on the observation that the rate limiting step is tunneling through the potential barriers represented by Eq. (2) [5]. This, together with $p_{0}$ and $p_{\nu}$ from Eq. (3), gives the scaling laws shown in Table \ for systems with one resonant pair of atoms. We show the results for $X Y Z$ systems (three distinguishable atoms) and for $B B X$ systems (two identical bosons and a third distinguishable atom). For $X Y Z$ systems, we have assumed that the $X-Y$ pair interacts resonantly and the $Z$ atom is in an nonresonant state. For $B B X$, we assumed that only the intraspecies interaction is resonant and, therefore, the $X$ atom is in an nonresonant state. Note that the scaling laws for both $X Y Z$ and $B B X$ systems are exactly the same. In the table, we show the first three partial wave contributions to vibrational relaxation of weakly bound molecules, $X Y^{*}+Z \rightarrow X Y+Z\left(V_{\text {rel }}\right)$; to three-body recombination into weakly bound molecules, $X+Y+Z \rightarrow X Y^{*}+Z$ $\left(K_{3}^{\mathrm{w}}\right)$; to recombination into deeply bound molecules, $X+Y+Z \rightarrow X Y+Z\left(K_{3}^{\mathrm{d}}\right)$; and to dissociation of weakly bound molecules, $X Y^{*}+Z \rightarrow X+Y+Z\left(D_{3}\right)$.

TABLE I: Energy and scattering length dependence for threebody collision rates for systems writh a single resonant interaction. Boldface indicates the leading contribution at threshold $\left(k \rightarrow 0\right.$ where $k=\sqrt{2 \mu E}$ for $K_{3}$ and $D_{3}$ and $k=\sqrt{2 \mu\left(E-E_{v}\right)}$ for $V_{\text {rel }},-E_{v}$ is the molecular binding energy). The results are valid for all three-body systems with three distinguishable atoms $(X Y Z)$ or with two identical bosons $(B B X)$ with $a_{B B}$ resonant.

\begin{tabular}{ccccccc}
\hline \hline & \multicolumn{2}{c}{$V_{\text {rel }}$} & & & $K_{3}^{\mathrm{w}}\left(D_{3}\right), K_{3}^{\mathrm{d}}$ & $K_{3}^{\mathrm{d}}$ \\
\cline { 2 - 3 } \cline { 5 - 7 }$J^{\pi}$ & $E$ & $a>0$ & & & \\
\hline $0^{+}$ & const & $\boldsymbol{a}^{-1}$ & $\operatorname{const}\left(\boldsymbol{k}^{4}\right)$ & $\boldsymbol{a}^{4}, \boldsymbol{a}^{2}$ & $|\boldsymbol{a}|^{2}$ \\
$1^{-}$ & $k^{2}$ & $a^{-1}$ & $k^{2}\left(k^{6}\right)$ & $a^{6}, a^{2}$ & $|a|^{2}$ \\
$2^{+}$ & $k^{4}$ & $a^{-1}$ & $k^{4}\left(k^{8}\right)$ & $a^{8}, a^{2}$ & $|a|^{2}$ \\
\hline \hline
\end{tabular}

Figure 1(a) shows our numerical results for $V_{\text {rel }}$, obtained from a direct solution of Eq. (11) (see Ref. 12] for details) for three distinguishable atoms, $X Y Z$, for $J^{\pi}=0^{+}(\pi$ is the total parity). We have assumed a finite-range model two-body interaction in which the resonant $X-Y$ interaction is adjusted to support one weakly bound $s$-wave state whose position controls the scattering length, one deeply bound $s$-wave state, and one deeply bound $p$-wave state. Our model $X-Z$ interaction sup- 
ports only one $s$-wave state, and, for simplicity, we assumed the $Y-Z$ interaction has no bound state. The figure shows that when $a / r_{0} \gtrsim 10$, our predicted $a^{-1}$ scaling law is verified by the numerical results. Thus, we have clear evidence that vibrational relaxation is indeed suppressed for large $a$ even in the absence of fermionic statistics. By extension, this result also support our arguments that molecular decay in $B B+X$ collisions are also suppressed as $a^{-1}$ even though bosonic statistics apply. In a two-component gas of $B$ and $X$ atoms, however, long lifetimes for $B B$ molecules can only be achieved if there are no free $B$ atoms and with the additional condition of low molecular density, to prevent decay due to molecule-molecule collisions.

Recombination for systems involving nonresonant atoms also presents some peculiar properties. As mentioned previously, for $a>0$, recombination into weakly bound molecules $\left(K_{3}^{\mathrm{w}}\right)$ scales as $a^{4}$ while recombination into deeply bound molecules $\left(K_{3}^{\mathrm{d}}\right)$ scales as $a^{2}$ (see Table I). Therefore, recombination for large $a$ leads mostly to the formation of weakly bound molecules. This differential scaling with $a$ is in stark contrast to previously studied systems with two or three resonant interactions for which recombination is non-zero at $T \rightarrow 0$, as is the case for one- and two-component bosonic systems and boson-fermion mixtures. In these systems, recombination into all two-body states scales as $a^{4}$. In those systems, recombination into the weakly bound state is typically dominant as well, but only via the coefficient of $a^{4}$. Systems with only a single resonant interaction share this property in addition to the differential scaling with $a$. Figure 1(b) shows our numerical calculations of recombination rates for the same system as in Fig. 1(a). The numerical results support all of the above predictions, including not only the smaller magnetude of $K_{3}^{d}$ relative to $K_{3}^{w}$ but also their scaling with $a$. Another consequence of this differential scaling is that for $a<0$, i.e., in the absence of weakly bound molecules, recombination is proportional to $|a|^{2}$. So, recombination at $a<0$ for systems with a single resonant pair could be suppressed compared to $a>0$ for large $a$.

The results obtained here for three-body processes involving a single resonant pair - summarized in Table I - combined with previous results [5] exhaust all possible three-body processes relevant to three-component atomic gases. The competition between the various collision processes in a multi-component gas is dictated fundamentally by their energy and scattering length dependence, although it is also crucial to take into account the atomic and molecular densities. In fact, manipulating the atomic and molecular densities can make favorable a particularly desired collisional behavior.

For completeness, we show in Table \the three-body collision rates for all possible ultracold three-component atomic gases. For each mixture, we have listed the threebody processes according to which interatomic interac-

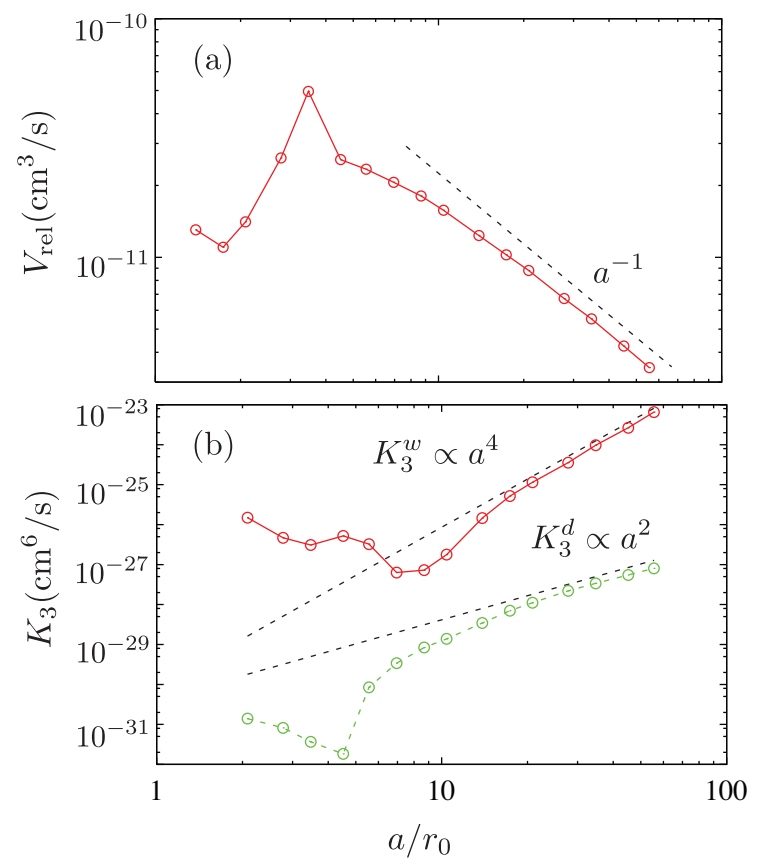

FIG. 1: (a) Vibrational relaxation rate from weakly bound $X Y^{*}$ molecules and (b) three-body recombination rates into weakly, $K_{3}^{\mathrm{w}}$, and deeply, $K_{3}^{\mathrm{d}}$, bound molecules.

tion is resonant by indicating the resonant pair of atoms, and we have only included the dominant contribution at threshold. Three-body processes involving a single resonant pair are indicated by " $\diamond$ " while all the others were obtained in previous work [4, 5, 7, ,9]. Futher, we have $2 \leq p_{0} \leq 4$ for $V_{\text {rel }}$ and $0 \leq p_{0} \leq 2$ for $K_{3}^{\mathrm{d}}$, depending on the mass ratio between the collision partners [5], and we have omitted modulation factors that produce the interference minima and resonant peaks related to Efimov physics [5].

We can see from the table that in all possible threecomponent mixtures weakly bound molecules can be expected to be long-lived with relaxation scaling as $a^{-1}$, provided that all free atoms are nonresonant. In fact, for gas mixtures where distinguishable fermions are resonant, weakly bound molecules are expected to be longlived irrespective of the identity of the free atoms.

Given the suppression possible in atom-molecule collisions, it might be that molecule-molecule collisions are the dominant relaxation mechanism in a threecomponent gas. For $B F$ molecules, that are themselves fermions, molecule-molecule collisions are suppressed at ultracold temperatures due to their $p$-wave character. For $B_{i} B_{j}$ bosonic molecules, however, long lifetimes can only be reached for low molecular densities, in order to prevent molecule-molecule collisions. In contrast, for $F_{i} F_{j}$ bosonic molecules, relaxation due to moleculemolecule collisions scales as $a^{-s}$ with $s>1$ [4] for mass ratios $m_{F_{i}} / m_{F_{j}}>0.11603$ (a condition satisfied for commonly used alkali atoms) and the main molecular colli- 
TABLE II: All three-body collision rates relevant for three-component atomic gases. For each mixture, the resonant pair of atoms are indicated by parentheses. The symbol " $\diamond$ " indicates collisions involving a single resonant interaction.

\begin{tabular}{|c|c|c|c|c|c|c|c|c|c|}
\hline Gas Mixture & & $V_{\mathrm{rel}}^{i j+k}$ & $K_{3}^{\mathrm{w}}, K_{3}^{\mathrm{d}}$ & & $V_{\mathrm{rel}}^{i j+k}$ & $K_{3}^{\mathrm{w}}, K_{3}^{\mathrm{d}}$ & & $V_{\mathrm{rel}}^{i j+k}$ & $K_{3}^{\mathrm{w}}, K_{3}^{\mathrm{d}}$ \\
\hline \multirow[t]{3}{*}{$B_{1}-B_{2}-B_{3}$} & $\left(B_{1} B_{1}\right) B_{1}$ & $a$ & $a^{4}, a^{4}$ & $\left(B_{1} B_{2}\right) B_{1}$ & $a$ & $a^{4}, a^{4}$ & & & \\
\hline & $\left(B_{1} B_{1}\right) B_{2}^{\diamond}$ & $a^{-1}$ & $a^{4}, a^{2}$ & $\left(B_{1} B_{2}\right) B_{2}$ & $a$ & $a^{4}, a^{4}$ & & & \\
\hline & $\left(B_{1} B_{1}\right) B_{3}^{\diamond}$ & $a^{-1}$ & $a^{4}, a^{2}$ & $\left(B_{1} B_{2}\right) B_{3}^{\diamond}$ & $a^{-1}$ & $a^{4}, a^{2}$ & & & \\
\hline \multirow[t]{3}{*}{$B_{1}-B_{2}-F$} & $\left(B_{1} B_{1}\right) B_{1}$ & $a$ & $a^{4}, a^{4}$ & $\left(B_{1} B_{2}\right) B_{1}$ & $a$ & $a^{4}, a^{4}$ & $\left(B_{1} F\right) B_{1}$ & $a$ & $a^{4}, a^{4}$ \\
\hline & $\left(B_{1} B_{1}\right) B_{2}^{\diamond}$ & $a^{-1}$ & $a^{4}, a^{2}$ & $\left(B_{1} B_{2}\right) B_{2}$ & $a$ & $a^{4}, a^{4}$ & $\left(B_{1} F\right) B_{2}^{\diamond}$ & $a^{-1}$ & $a^{4}, a^{2}$ \\
\hline & $\left(B_{1} B_{1}\right) F^{\diamond}$ & $a^{-1}$ & $a^{4}, a^{2}$ & $\left(B_{1} B_{2}\right) F^{\diamond}$ & $a^{-1}$ & $a^{4}, a^{2}$ & $B_{1} F F$ & $a^{1-2 p_{0}}$ & $k^{2} a^{6}, k^{2} a^{6-2 p_{0}}$ \\
\hline \multirow[t]{3}{*}{$B-F_{1}-F_{2}$} & $(B B) B$ & $a$ & $a^{4}, a^{4}$ & $\left(B F_{1}\right) B$ & $a$ & $a^{4}, a^{4}$ & $\left(F_{1} F_{2}\right) B^{\diamond}$ & $a^{-1}$ & $a^{4}, a^{2}$ \\
\hline & $(B B) F_{1}^{\diamond}$ & $a^{-1}$ & $a^{4}, a^{2}$ & $\left(B F_{1}\right) F_{1}$ & $a^{1-2 p_{0}}$ & $k^{2} a^{6}, k^{2} a^{6-2 p_{0}}$ & $\left(F_{1} F_{2}\right) F_{1}$ & $a^{1-2 p_{0}}$ & $k^{2} a^{6}, k^{2} a^{6-2 p_{0}}$ \\
\hline & $(B B) F_{2}^{\diamond}$ & $a^{-1}$ & $a^{4}, a^{2}$ & $\left(B F_{1}\right) F_{2}^{\diamond}$ & $a^{-1}$ & $a^{4}, a^{2}$ & $\left(F_{1} F_{2}\right) F_{2}$ & $a^{1-2 p_{0}}$ & $k^{2} a^{6}, k^{2} a^{6-2 p_{0}}$ \\
\hline \multirow[t]{3}{*}{$F_{1}-F_{2}-F_{3}$} & $\left(F_{1} F_{2}\right) F_{1}$ & $a^{1-2 p_{0}}$ & $k^{2} a^{6}, k^{2} a^{6-2 p_{0}}$ & & & & & & \\
\hline & $\left(F_{1} F_{2}\right) F_{2}$ & $a^{1-2 p_{0}}$ & $k^{2} a^{6}, k^{2} a^{6-2 p_{0}}$ & & & & & & \\
\hline & $\left(F_{1} F_{2}\right) F_{3}^{\diamond}$ & $a^{-1}$ & $a^{4}, a^{2}$ & & & & & & \\
\hline
\end{tabular}

sional decay is due to atom-molecule collisions involving a single resonant interaction, scaling as $a^{-1}$.

From Table we see that the optimal three-component systems utilize molecular formation close to an $F_{1}-F_{2}$ Feshbach resonance. In this case, recombination involving two identical fermions is suppressed at ultracold temperatures while recombination in both $B-F_{1}-F_{2}$ and $F_{1}$ $F_{2}-F_{3}$ mixtures leads mainly to the formation of weakly bound $F_{1} F_{2}$ molecules. The most important property of this system is that after the weakly bound molecules are formed, molecular loss due to collisions with the remaining atoms is suppressed at least as $a^{-1}$. Similar benefits can also be realized near a $B-F$ resonance under certain circumstances. For instance, in $B-F_{1}-F_{2}$ mixtures, efficient $B-F_{1}$ molecular formation could be achieved if the number of nonresonant $F_{2}$ atoms is higher than the $B$ and $F_{1}$ numbers. If in the end all bosons are bound in $B F$ molecules, then collisions with the remaining fermions is suppressed, ensuring the desired stability of weakly bound molecules.

To summarize, we have shown that vibrational relaxation of weakly bound molecules can be suppressed near a Feshbach resonance even in the absence of Fermi statistics. Remarkably, the root cause of this suppression can be traced to the same kind of universality that yields the Efimov effect. The systems that show this behavior can also exhibit differential scaling with $a$ of the recombination rate into weakly bound molecules that is finite at zero temperature. These two results combine to make ultracold three-component gases with a single resonant interaction an attractive alternative for efficiently producing stable, ultracold molecules.

This work was supported by the National Science Foundation, the Air Force Office of Scientific Research, and by the W. M. Keck Foundation.
[1] M. Greiner, C. A. Regal, and D. Jin, Nature (London) 426, 537 (2003); J. Cubizolles et al., Phys. Rev. Lett. 91, 240401 (2003); C. A. Regal, M. Greiner, and D. S. Jin Phys. Rev. Lett. 92, 083201 (2004); S. Jochim et al., Science 3022101 (2003); M. W. Zwierlein et al., Phys. Rev. Lett. 92, 120403 (2004); K. E. Strecker, G. B. Partridge, and R. G. Hulet, ibid., 91080406 (2003).

[2] C. A. Regal, M. Greiner, and D. S. Jin, Phys. Rev. Lett. 92, 040403 (2004); M. W. Zwierlein et al., ibid. 92, 120403 (2004); C. Chin et al., Science 305, 1128 (2004); M. W. Zwierlein, J. R. Abo-Shaeer, A. Schirotzek, C. H. Schunck, and W. Ketterle, Nature (London) 435, 1047 (2005).

[3] J. J. Zirbel, K.-K. Ni, S. Ospelkaus, J. P. D'Incao, C. E. Wieman, J. Ye, and D. S. Jin, condmat.other $/ 0710.2479 v 1$

[4] D. S. Petrov, C. Salomon, and G. V. Shlyapnikov, Phys. Rev. Lett. 93, 090404 (2004); J. Phys. B 38, 645 (2005).

[5] J. P. D'Incao and B. D. Esry, Phys. Rev. Lett. 94, 213201 (2005).

[6] V. A. Yurovsky, A. Ben-Reuven, P. S. Julienne, and C. J. Williams, Phys. Rev. A 60, R765 (1999).

[7] E. Braaten and H.-W. Hammer, Phys. Rev. A 70, 042706 (2004).

[8] J. P. D'Incao, H. Suno, and B. D. Esry, Phys. Rev. Lett. 93, 123201 (2004); J. P. D'Incao, and B. D. Esry, Phys. Rev. A 73, 030702(R) (2006); cond-mat/0703269.

[9] E. Braaten and H.-W. Hammer, Phys. Rep. 428, 259 (2006).

[10] T. Weber, et al., Phys. Rev. Lett. 91, 123201 (2003).

[11] J. Cubizolles, et al., Phys. Rev. Lett. 91, 240401 (2003); S. Jochim, et al., Phys. Rev. Lett. 91, 240402 (2003); M. W. Zwierlein, et al., Phys. Rev. Lett. 91, 250401 (2003).

[12] H. Suno, B. D. Esry, C. H. Greene and J. P. Burke, Jr., Phys. Rev. A 65, 042725 (2002). 